\title{
Metamodelo del sistema de regulación de la demanda de agua potable en horizontes de largo plazo
}

\author{
Drinking water demand regulation system metamodel \\ in long term horizons \\ José Andelfo Lizcano Caro ${ }^{1 *} \quad$ Sandro Javier Bolaños ${ }^{2} \quad$ Rubén Javier Medina Daza ${ }^{2}$ \\ Recibido 28 de septiembre de 2017, Aceptado 20 de agosto de 2018 \\ Received: September 28, 2017 Accepted: August 18, 2018
}

\begin{abstract}
RESUMEN
La regulación para agua potable, a nivel global, se cimienta en costos de prestación y demanda futura. Las empresas buscan flexibilidad financiera; los suscriptores, eficiencia. La suficiencia financiera se centra en proyecciones de costos y tasas de descuento que remuneren las inversiones; la eficiencia económica, en reducir algunos puntos y emitir señales que eviten sobredimensionamientos y controlen cuellos de botella. Mientras, variables como valor presente de la demanda en horizontes de largo plazo (HVPD), han quedado al margen de una regulación que no ha sido capaz de responder a la inestable demanda de agua y a fenómenos ambientales como el cambio climático. Para responder a estas exigencias y a las distorsiones socioeconómicas regionales, dentro de un proyecto de investigación científica, se propone la creación de un metamodelo para regulación del sector de agua potable, en escenarios de equilibrio inestable, que considere criterios de solidaridad y oriente la priorización de inversiones ecoambientales.
\end{abstract}

Palabras clave: Demanda de agua potable, metamodelo, sistema de regulación.

\begin{abstract}
A global level, the regulation for the provision of the aqueduct service is based on the costs of providing the company, but above all on future demand, i.e. the main indicator to calculate the costs and costs of production of the good and service. Efficiency, financial sufficiency, and budget depend on the accuracy of measurements and projections for a market environment. Financial sufficiency focuses on projections of costs and discount rates that remunerate investments. Economic efficiency, reducing oversizing in operational as well as administrative terms and control bottlenecks. Meanwhile, variables such as the present value of demand in long-term horizons (HVPD) have been left out of a regulation that had not been able to respond to unstable water demand and environmental phenomena such as climate change. To respond to these demands and regional socio-economic distortions, within a scientific research project, it is proposed to create a meta-model for the regulation of the potable water sector, in unstable equilibrium scenarios, considering criteria of solidarity and orient Prioritization of eco-environmental investments.
\end{abstract}

Keywords: Drinking water demand, metamodel, regulation system.

1 Universidad Distrital Francisco José de Caldas. Facultad de Medio Ambiente y Recursos Naturales. Bogotá, Colombia. E-mail: jalizcanoc@udistrital.edu.co

2 Universidad Distrital Francisco José de Caldas. Facultad de Ingeniería. Bogotá, Colombia. E-mail: bsandrojavier@ gmail.com; rmedina@udistrital.edu.co

* Autor de correspondencia: jalizcanoc@udistrital.edu.co 


\section{INTRODUCCIÓN}

El manejo del agua urbana involucra los campos de suministro de agua, drenaje urbano, tratamiento de aguas residuales y manejo de lodos. Sobre la base del encuentro Agenda 21, se discuten los principios y directrices para la gestión sostenible del agua urbana. La tecnología sostenible conduce a gradientes aceptables en las variables de estado. Un uso eficiente de los recursos dará lugar a un aumento mínimo de la entropía y requerirá un enfoque activo en lugar de reactivo. El análisis del período de transición de la situación actual a una sostenible es importante para la toma de decisiones en la agenda administrativos y normativos [1].

Colombia ha venido trabajando en modelos teóricoprácticos para poder proveerse de herramientas técnicas que mejoren la toma de decisiones, sin embargo, los modelos estocásticos son los que han prevalecido en los últimos años. La proyección de demanda de agua potable ha sido parte de las herramientas científicas empleadas por las instituciones, sin embargo, no hay un consenso desde el Estado frente a cuál es la manera más apropiada de administrar el recurso hídrico en el largo plazo.

Una eficiente prestación de servicios públicos como el de agua potable [2], debe ser producto de una adecuada combinación de señales económicas, ambientales, técnicas y sociales [3]. Entre ellas, el grado de simetría de los criterios tarifarios [4]; la capacidad de los sistemas y su flexibilidad operativa, modelando de esta forma la disponibilidad de los servicios en el corto, mediano y largo plazo [5]. Para diseñar obras y proyectar su financiación, se requiere que, tanto prestadores como consumidores, conozcan la demanda esperada de los servicios [6], la cual a su vez está orientada por la estructura de costos y sus índices tarifarios, junto con la porción de la oferta a subsidiar [7-8]. Hay que considerar, también, estimaciones de las pérdidas comerciales y técnicas de agua [9].

Los países requieren de estudios que permitan trazar distintos rangos de consumo para planear la asignación de recursos, tanto regionales como nacionales, y definir metas e indicadores de inversión, de reducción de pérdidas y de disponibilidad en fuentes. También, investigar los niveles básicos de servicio que garanticen la subsistencia de los habitantes, considerando factores sociales y ecoambientales [10].
Lo anterior permitirá generar respuestas a requerimientos universales que exigen garantizar la atención de las comunidades con servicios esenciales en los niveles requeridos, sin afectar los criterios de suficiencia financiera y económica.

En este paper se expone, dos de los principales modelos regulatorios de agua potable en el mundo; luego, se desarrollan las bases para estructurar un metamodelo que responda de manera integral a un sistema de regulación y política sectorial [11], que entre otros aspectos, sirva para determinar escenarios posibles del consumo futuro de agua, que permitan estructurar estudios que se faciliten a las necesidades regulatorias de cada región. Su pertinencia es útil, especialmente, para países con regulación deficiente o problemas estructurales por la falta de fuentes para abastecer a las poblaciones; y con serias dificultades para soportar los volúmenes requeridos por la demanda derivada del crecimiento urbano [12-13].

\section{ANÁLISIS TEÓRICO}

Los metamodelos tienen una utilidad técnica y administrativa importante en programas como la administración integrada del recurso hídrico, compuestos de tres ejes [14]:

1) un entorno propicio de políticas y legislación adecuadas sobre recursos hídricos.

11) un marco institucional de instituciones capaces a nivel nacional, local y de cuenca fluvial.

111) Un conjunto de instrumentos de gestión para estas instituciones.

Los cuales pueden ser un buen reemplazo a esfuerzos aislados plasmados en planes del agua y programas.

En el entorno colombiano, la Corporación Autónoma Regional (CAR) es la entidad estatal responsable de estudiar, regular y promover políticas que tengan consecución en la administración responsable del recurso hídrico.

La CAR se apoya en instituciones como el IDEAM (Instituto de Hidrología, Meteorología y Estudios Ambientales), INSTITUTO VON HUMBOLDT es responsable de investigación biológica y en biodiversidad, el INVEMAR, quien coordina estudios marinos y costeros, el Instituto Amazónico 
de Investigaciones Científicas, SINCHI, el Instituto de Investigaciones Ambientales del Pacífico - IIAP, entre otros [15].

\section{Modelos regulatorios representativos}

Se exponen dos de los esquemas regulatorios de mayor aplicación en sistemas capitalistas, que son el inglés y chileno operando desde supuestos de competencia y eficiencia; luego, se presenta el modelo colombiano [16].

\section{Chile. Regulación por Empresa Modelo (Eficiencia absoluta)}

La regulación en Chile ha seguido el esquema de empresa modelo. La Superintendencia de Servicios Sanitarios es la encargada de regular y fiscalizar las tarifas para los servicios de acueducto, alcantarillado, otorgar concesiones y controlar residuos industriales líquidos.

Se basa en el principio de eficiencia dinámica bajo el cual se establecen las tarifas en forma independiente de los costos reales, buscando el punto de equilibrio entre la oferta y la demanda. La empresa real debe ajustar sus costos en busca de este nivel. Cada vez que se fijan tarifas, se involucran mejoramientos de productividad, experimentados en la prestación del servicio. La empresa modelo se diseña con base en los servicios requeridos por la población, la normatividad, las restricciones geográficas, demográficas y tecnológicas en las que se enmarca su operación [17].

El precio se define con base en el principio de eficiencia económica, buscando fijar el precio con base el costo de oportunidad de producir una unidad adicional del bien, es decir con el costo marginal: Costo Incremental de Desarrollo (CID).

\section{Modelo de regulación Reino Unido (Privatización) La OFWAT (Office of Water Services), entidad reguladora de los servicios de acueducto y alcantarillado del Reino Unido, establece sus criterios de protección al consumidor estableciendo precios techo (price caps), revisa y aprueba tarifas, controla e incentiva el mercado de agua potable de empresas prestadoras en Inglaterra y Gales.}

Los límites de precios se establecen cada cinco años, hay un mecanismo de penalización e incentivos para las empresas, de acuerdo al rendimiento. Los criterios para el establecimiento de las tarifas incluyen: $\checkmark \quad$ Ser justos entre los diferentes grupos de clientes (sería interesante profundizar aquí o en otro aparte sobre este concepto de justicia).

$\checkmark$ Fomentar el uso racional del agua.

$\checkmark$ Tener en cuenta los bajos ingresos de los clientes domésticos.

$\checkmark$ Ser razonablemente estable evitando al máximo los cambios.

La regulación se basa en benchmarking (también llamado Yardstick competition) o competencia por comparación. A cada empresa regulada, i, se le asigna una empresa sombra que sirve de referencia para determinar las necesidades de ingresos.

Esta regulación es plasmada en el denominado "libro blanco" o "Water White Paper", cuyo objetivo es aumentar la competencia en el sector del agua [18], pero éste solo se realizará si los nuevos participantes en la industria pueden competir de manera efectiva con las empresas monopolizadoras del agua. Parte de la experiencia que dejó la Ley de Agua de 2003, la cual no introdujo una competencia significativa para el sector, por tanto se ha atribuido en parte a las barreras legales que enfrentan los nuevos participantes y las dificultades prácticas que implican tratar de obtener negocios de los titulares.

El informe del Profesor Martin Cave [19] sobre Competencia e Innovación en los Mercados del Agua consideró una gama de opciones para introducir una competencia efectiva en el sector del agua. Llegando a la conclusión de que, dadas las dificultades que enfrentan los nuevos competidores para competir con las empresas establecidas, el gobierno debería ordenar la separación legal de las operaciones minoristas de los incumbentes del resto de sus negocios, minimizando así la probabilidad de discriminación injusta y alentando a nuevos participantes en el mercado.

\section{Modelo regulador colombiano (Libertad regu- lada) [20]}

El modelo de regulación parte de un deber de las autoridades municipales, las cuales deben garantizar el acceso de agua potable y saneamiento a su población. Sus responsabilidades van desde la extracción de agua, almacenamiento, distribución y descarga, incluido el tratamiento de aguas residuales. Usualmente otorgan la concesión para la operación del servicio de agua potable y saneamiento a una 
entidad o utilidad especializada, que puede ser privada, semiprivada o pública. Cada empresa debe tener un Plan Maestro, que incluye las principales inversiones para la expansión de la cobertura de agua potable y saneamiento en las áreas urbanas [21].

La inversión y operación se financia principalmente con tarifas a los usuarios. Las tarifas están subsidiadas para usuarios de bajos ingresos, mientras que los usuarios de altos ingresos tienen que contribuir con costos operativos superiores a la media. En la mayoría de los municipios, los subsidios son más altos que las contribuciones $y$, por lo tanto, el déficit debe cubrirse mediante transferencias del gobierno nacional a los municipios. El cálculo de las tarifas está regulado por un Comité Nacional de Regulación del Agua Potable. Las tarifas deben recuperar completamente los costos de operación y recuperar parcialmente los costos de inversión del servicio. El Comité ha aprobado solo la inclusión de costos ambientales o inversiones en las tarifas limitadas a los cargos requeridos por la ley (cargos por agua y contaminación). El Ministerio del Ambiente diseña la política nacional de agua potable y servicios domésticos y ayuda a las municipalidades a alcanzar sus metas de aumentar la cobertura en agua potable y saneamiento. El Ministerio cofinancia inversiones en agua potable y saneamiento con préstamos multilaterales y dinero proveniente de los ingresos públicos [22].

Las CAR también cofinancian sistemas de tratamiento de aguas residuales con los ingresos por la carga contaminante.

El acceso o expansión de la toma de agua para el sistema de agua potable requiere un permiso de agua otorgado por el CAR con la jurisdicción correspondiente. Para otorgar un permiso de agua, el CAR analizará la disponibilidad del recurso y su impacto en otros usuarios. La ley ambiental otorga prioridad a la asignación de agua para usos domésticos. Una vez que se expide el permiso de agua, la empresa de servicios públicos puede acceder o ampliar la admisión y deberá pagar la tarifa de agua al CAR [23].

\section{El caso de Bogotá [24]}

El costo de referencia de un metro cúbico de agua potable en Bogotá asciende a cerca de un dólar americano. Verterlo cuesta 0,80 centavos de dólar.
Un suscriptor de estrato bajo-bajo o 1, recibe un subsidio del $70 \%$. Al considerar que el rango de consumo subsidiable va hasta 20 metros cúbicos al mes y que los estratos bajo y medio bajo también reciben subsidios -en proporciones del $40 \%$ y $15 \%$ - el monto de subsidios en Bogotá supera los US 80 millones de dólares al año.

Si el rango de agua que se considera necesario para subsistir se redujera, el resultado negativo entre asignaciones, subsidios y sobreprecios, sería menos deficitario. Pero esta consideración tendría que ser evaluada bajo el criterio de una demanda relativamente inelástica al precio (60). Ahora, si parte del impacto social fuese absorbido por mecanismos de uso eficiente del agua, en términos de la preservación de los recursos naturales la señal sería positiva. Ello requeriría estimar márgenes de posibles ahorros de agua, con efectos en los períodos tarifarios.

Exámenes como los referidos son una parte de las variables, consideraciones, análisis y evaluaciones que deben adelantarse para dar respuesta al mandato constitucional que señala que el precio de los servicios públicos debe considerar criterios de solidaridad y redistribución de ingresos, permitiendo a las poblaciones de menores ingresos tener acceso a cantidades de servicios que satisfagan sus necesidades básicas.

El régimen de los servicios públicos, le dio la competencia a las comisiones de regulación para que definieran la línea de consumo hasta donde se asignarían subsidios [25]. La Comisión de Regulación de Agua Potable y Saneamiento Básico -CRA-, ha mantenido el consumo básico en 20 metros cúbicos al mes y el complementario en el doble de ese volumen.

Después de casi 20 años, la CRA no ha podido regular este tema, sensible como es tanto al pago de los servicios como a la capacidad de los sistemas y a la preservación de los recursos naturales.

\section{Desarrollo del Metamodelo}

Para la propuesta del metamodelo de regulación del sistema de agua potable, se parte de la definición de los problemas regulatorios, planeando una solución desde el equilibrio; luego, se definen las leyes, axiomas, principios y criterios orientadores, 
dentro de un modelo de secuencia de prestación. Con la detección de los principales índices y variables de suministro de los servicios, se exponen nuevos escenarios de prestación, valiéndonos de robustos indicadores de gestión y resultados. De la investigación en distintos esquemas de regulación, se exponen los principales modelos que dan respuesta a problemas clásicos de la prestación y a nuevos problemas que hacen más incierta la regulación y respuesta a demandas de agua. El equilibrio de estos modelos, y sus conectores, sustentan el metamodelo que ofrece respuestas a distintos escenarios de prestación.

\section{Equilibrio regulatorio, como respuesta al problema} La carencia de un sistema integral, bajo regímenes de libertad, para determinar regulaciones para la demanda de agua en horizontes de largo plazo, que integren varios de los modelos existentes y los haga más robustos y capaces de dar respuesta a fenómenos como el cambio climático, el crecimiento urbano sin orden territorial y la contaminación de las aguas y el agotamiento de las fuentes naturales, es el problema que debe resolver el metamodelo para regulación del agua.
En la Figura 1 se muestra el problema, trazado desde la analogía del equilibrio, como base de un metamodelo de solución.

La definición adecuada de indicadores de gestión y resultados [27-29] serán la base para que el metamodelo refleje el comportamiento real de la creciente demanda de agua [30-31]. Una vez obtenidos, los indicadores alimentarán sistemas matriciales que se modelarán en períodos de proyección [32-33].

\section{Categorizaciones}

Aquí se describen las leyes, principios, axiomas, criterios y supuestos que soportan el metamodelo para el sistema regulatorio propuesto, los cuales se describen a continuación y se representan en la Figura 2.

\section{Leyes:}

i. Las reducciones en el índice de agua no contabilizada -IANC-, comprimen el valor presente de la demanda-VPD- [3].

ii. Las economías de escala siempre son verificables en el suministro de agua [34].

Fuente. [26]

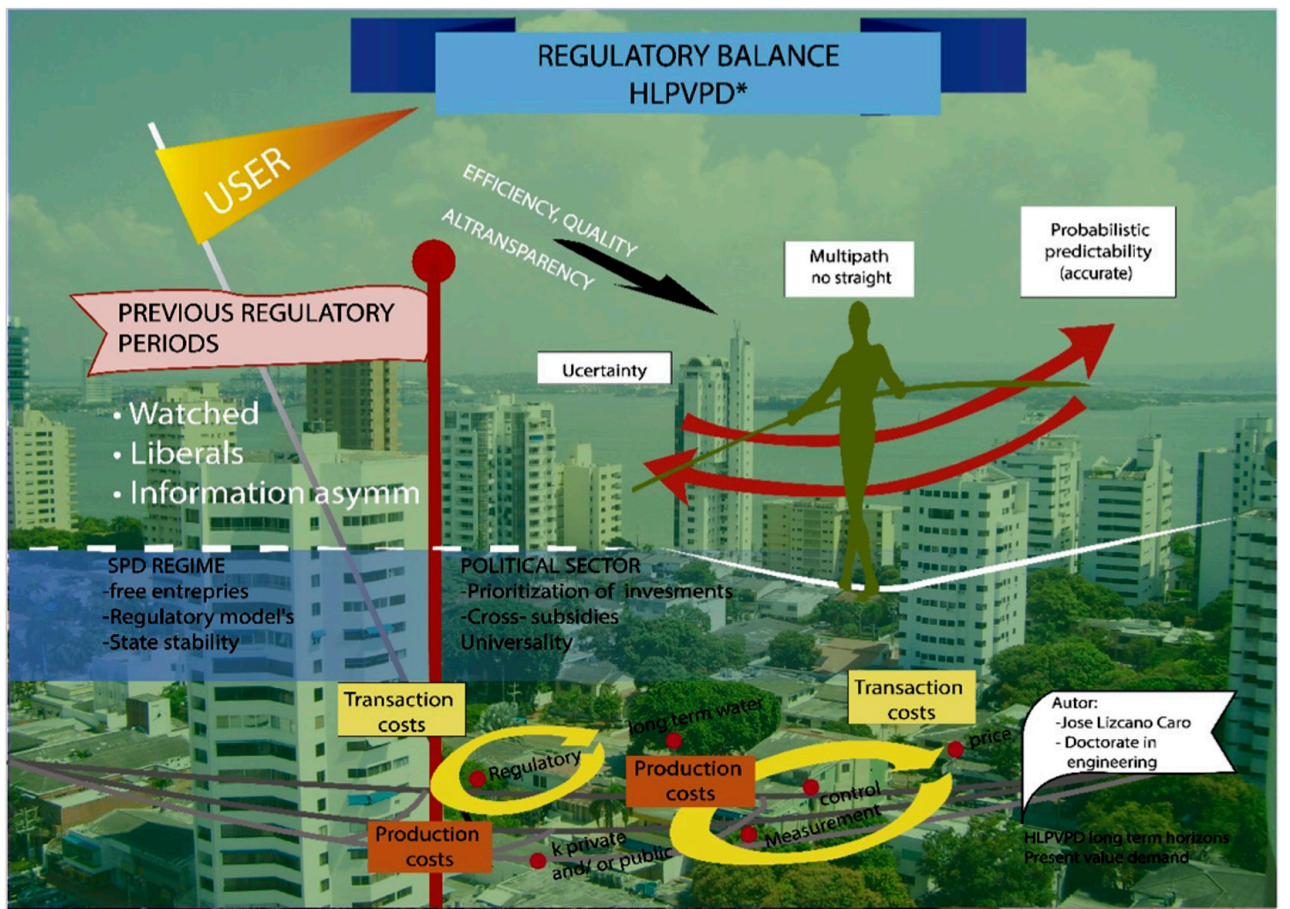

Figura 1. Representación del balance regulatorio en horizontes de proyección a largo plazo. 
Axiomas:

i. La oferta y demanda de agua potable configura mercados imperfectos cuya rentabilidad debe definirla el regulador mediante rangos que reflejen dicha imperfección.

ii. El agua es un bien relativamente escaso orientado a satisfacer necesidades vitales.

iii. Los costos de su proceso se dividen entre costos de producción (o transformación a la potabilidad) y costos de transacción (o de agente hacia su puesta en uso al suscriptor).

\section{Principios:}

i. Críticas al principio de que el agua es un recurso natural renovable [35].

ii. Racionalidad. El manejo del recurso hídrico se fundamenta en el uso racional y eficiente [36].

iii. Vida útil. La de los activos asociados a la infraestructura es función del horizonte de planeación de la demanda futura [37].

\section{Criterios:}

i. Suficiencia financiera y eficiencia económica [38].

ii. Solidaridad y redistribución de ingresos [38].

iii. Neutralidad.
Supuestos:

i. Uso racional.

ii. Vida útil de activos -f(HPVD).

\section{Secuencia del modelo de prestación propuesto}

El esquema típico de prestación, está influenciado por la alta intrusión estatal, con baja transparencia en el modelo de regulación y prestación; asignado con una planeación y gestión por vía presupuestal, donde las juntas directivas de entidades estales distorsionan la expansión, la eficiencia y la calidad del servicio; con criterios de política, regulación y control sin criterios uniformes; con asimétricos sistemas de información, basado en datos aportados, de manera dispersa, por los mismos prestadores; carentes de un sistema central de información; con esfuerzos regulatorios enfocados a establecer topes tarifarios; con un sistema de vigilancia y control común a cualquier entidad del orden descentralizado; y con usuarios desprotegidos y sin instancias de apelación y con límites jurídicos para su organización.

El modelo de prestación de los servicios públicos domiciliarios de agua potable, determinado para la investigación, se define en el siguiente diagrama de secuencia.

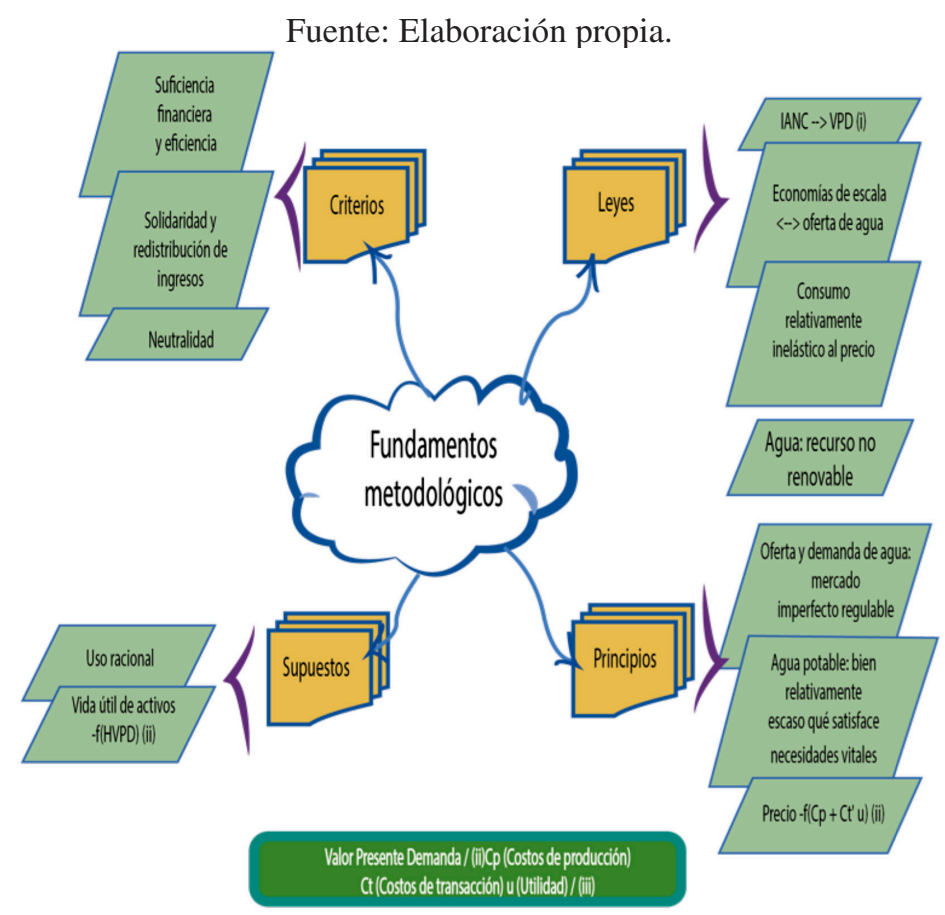

Figura 2. Fundamentos metodológicos que soportan el metamodelo para el sistema regulatorio propuesto. 


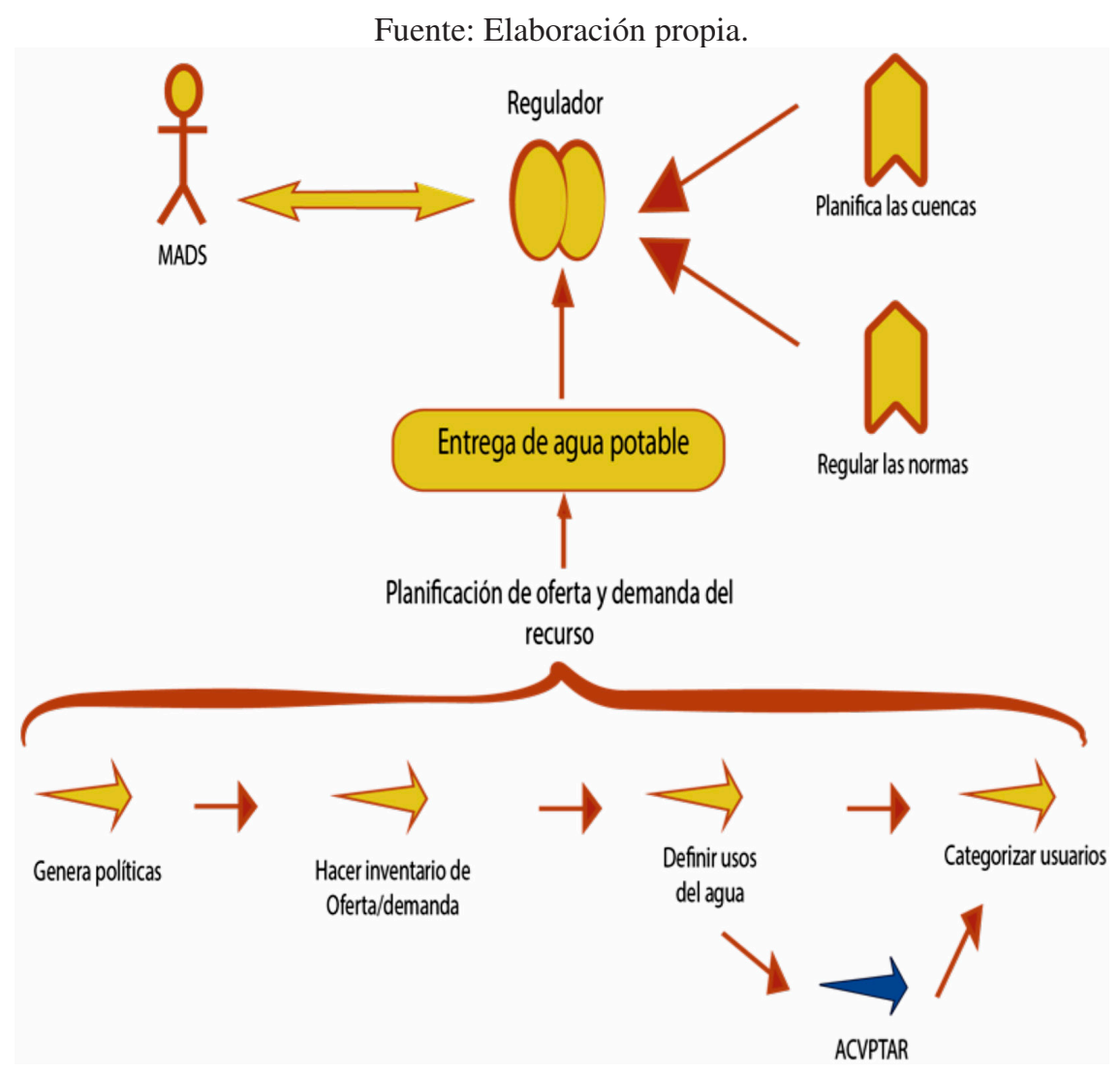

Figura 3. MADS: Ministerio encargado de trazar la política sectorial.

En la Figura 3 se muestra el modelo de secuencia de prestación en el cual se explica el esquema típico de prestación.

\section{Indicadores del Metamodelo}

Lo expuesto, conduce a sustentar la importancia del asunto tratado en este artículo [39-41]. El metamodelo propuesto incluye indicadores sobre la escasez del recurso hídrico [42], la capacidad de las micro cuencas [43], el clima, la altura, la densidad poblacional, el peso en la canasta familiar [44], los hábitos de consumo y la capacidad instalada, entre otros [45]. También se considerarían los efectos sobre el servicio de alcantarillado y las estructuras de costos de operación, inversión y de tasas ambientales [37].

La incorporación de los costos de transacción (o de agente), consolidaría su pertinencia. Está probado que la precariedad institucional (en este caso las distorsiones que genera la regulatoria sectorial) incida de forma determinante en la formación de los precios en países de Tercer Mundo (3). Razón por la cual la tarifa conviene que desagregue los costos de agente que, además del objetivo regulador, incorporen las fragilidades del sistema oferta-demanda y las dificultades para medir los atributos y para hacer cumplir los contratos de prestación del servicio, una de ellas explicada en la generación permanente de conflictos entre prestador-suscriptor.

Para una eficiente prestación de servicios públicos, como el de agua, es fundamental una adecuada combinación de señales económicas, técnicas y sociales [46-47]. Entre otras, las de criterios tarifarios, la capacidad de los sistemas y su operación y la disponibilidad de los servicios. Para los diseños de obras y proyecciones financieras, tanto del ente prestador, como del consumidor, se requiere conocer la demanda esperada de los servicios [48], la cual a su vez está orientada por la estructura de costos y sus índices tarifarios junto con la oferta de las cantidades a subsidiar [49-51]. Ha de considerarse, también, el efecto de 
las pérdidas comerciales y técnicas tanto de agua potable como de agua cruda [9].

El metamodelo induciría a realizar estudios que tracen distintos rangos de consumo para planear la asignación de recursos, definir metas e indicadores de inversión y las de reducción de pérdidas y de disponibilidad en fuentes [52-54]. Dichos estudios han de investigar, de manera integral, los niveles básicos de servicio que garanticen la subsistencia de los habitantes considerando componentes sociales, ecológicos y ambientales [55]. Ello contribuiría a atender requerimientos de las comunidades con servicios esenciales, en los niveles requeridos [56-57].

El metamodelo opera bajo criterios exploratorioinductivos. Partirá de evaluar las estructuras de costos y su incidencia en la formación de tarifas cruzadas con la variable demanda de agua en horizontes de largo plazo y los rangos de consumo regulados (oferta y demanda) [58].

El estado del arte debe incorporar, con profundo rigor, la revisión de componentes técnicos y sociales de este tipo:

$\checkmark$ Costos de referencia de largo plazo [59].

$\checkmark$ Costos incrementales para consumos excesivos.

$\checkmark$ Horizontes de planeación de inversiones [60].

$\checkmark$ Horizontes de tiempo para proyectar demanda [61].

$\checkmark$ Volúmenes de agua producida.

$\checkmark$ Índices de agua no contabilizada.

$\checkmark$ Volúmenes de agua facturada.

$\checkmark$ Tamaño de mercados.

$\checkmark$ Estratificación de mercados.

$\checkmark$ Demandas de agua por estratos y usos.

$\checkmark$ Inversiones ambientales para reforestación y recuperación de cuencas.

$\checkmark$ Costos, impactos y tasas ambientales.

Esta información, criticada, sistematizada y modelada servirá para generar indicadores de este tipo:

$\checkmark$ Calidad y abundancia ecológica y su efecto en índices de escasez.

$\checkmark$ Correlación Demanda-Clima.

$\checkmark$ Demandas históricas de consumo [62].

$\checkmark$ Relación estrato vs consumo [63].

$\checkmark$ Elasticidad precio-demanda (apuntando a inelasticidad relativa).

$\checkmark$ Pérdidas de servicio.
Estos indicadores, permitirán diseñar y operar uno o varios modelos para calibrar uno o varios rangos de consumo básico, complementario y suntuario en horizontes de largo plazo.

A esta propuesta, como resultados parciales de investigación se le(s) podrían incorporarán otras posibles variables que puedan mejorar el sistema y arrojar mejores resultados [64].

El mecanismo propuesto permitiría crear la ontología para determinar consumos de agua potable en horizontes de largo plazo, desde distintos modelos [65], cruzando parámetros de eficiencia y escasez de recursos hídricos [66] junto con los de uso racional y eficiente del agua apta para consumo humano.

De allí, se derivarían insumos para diseñar uno o varios modelos que enriquecieran de mayor rigor la toma de decisiones regulatorias. Igualmente serían útiles para que las empresas del sector proyecten sus demandas de agua y las inversiones que les garanticen la captación, tratamiento, conducción y dotación del servicio a sus usuarios. Podría ocurrir que los impactos se dividieran entre los empresariales y los regulatorios.

\section{Los modelos del Metamodelo}

Los problemas reales del sector de agua potable y saneamiento básico, estructurados para la presente investigación, se exponen en la siguiente arquitectura de rueda. En un siguiente nivel, para la solución parcial de uno o varios problemas, se han definido los siguientes modelos, contenidos en la Figura 4.

En la Figura 4 se observa los modelos incluidos en el metamodelo, identificando los problemas detectados a solucionar por medio del metamodelo. Así, la solución de los problemas detectados, se resuelven con el Metamodelo expuesto.

Una vez armado el metamodelo, podría programarse para operar utilizando parámetros operativos sobre escenarios regidos por el principio de incertidumbre y/o la predictibilidad probabilística y/o multi trayectorias lineales no rectas, todo ello dentro de un marco teórico práctico de equilibrio inestable.

\section{RESULTADOS}

Con el problema de secuencia de prestación determinado; las categorías de leyes, axiomas, 


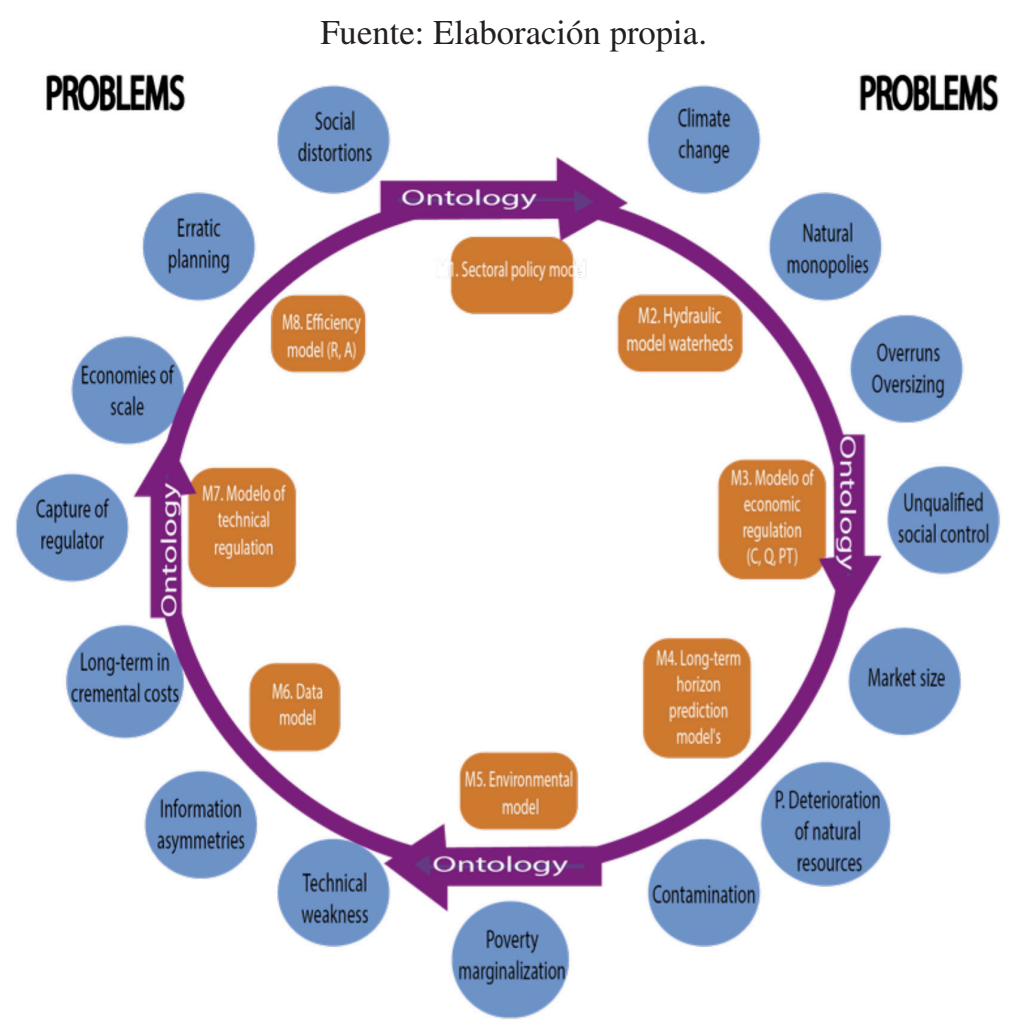

Figura 4. Problemas detectados a solucionar en la construcción de un metamodelo.

principios y criterios; los indicadores de gestión y resultados y las variables y modelos y conectores y transformadores, se llega a la propuesta del siguiente Metamodelo, expuesto en la Figura 5.

En la Figura 5 se observa un paper en el cual se obtuvieron los resultados la construcción del concepto metamodelo de regulación para agua en horizontes de planeación a largo plazo en valor presente. Ello, a partir de modelos sectoriales de política, de demandas, de eficiencia y, principalmente, el de equilibrio. Igualmente, se expusieron estudios de caso para diferentes latitudes del planeta.

\section{Posibles aplicaciones}

Algunas de las aplicaciones del Metamodelo se muestran en los siguientes tres (3) casos, expuesto de manera sintética; al igual que una aplicación concreta para la regulación colombiana:

\section{Caso 1}

En la regulación vigente se observa que las variables de consumos básico, complementario y suntuario no se han desarrollado mediante estudios matemáticos o estadísticos arriesgando que sus rangos queden afectados por consideraciones subjetivas.

\section{Caso 2}

En relación con la fijación de topes de medida para determinar o promover el uso eficiente y ahorro de agua potable $\mathrm{y}$, por ende, los denominados "consumos excesivos".

Caso 3

En relación con las pérdidas técnicas y comerciales, se formulan las siguientes glosas:

a) Niveles aceptables de pérdidas (comerciales (x medición o facturación), técnicas (x fugas y similares), en máximos del $30 \%$ cuyo monto no tendría sustentación empírica adecuadamente documentada.

b) El Instituto del Banco Mundial estableció un sistema de bandas de aproximación simplificada que permite, con base en el nivel de agua perdida por conexión, establecer unas metas de reducción 


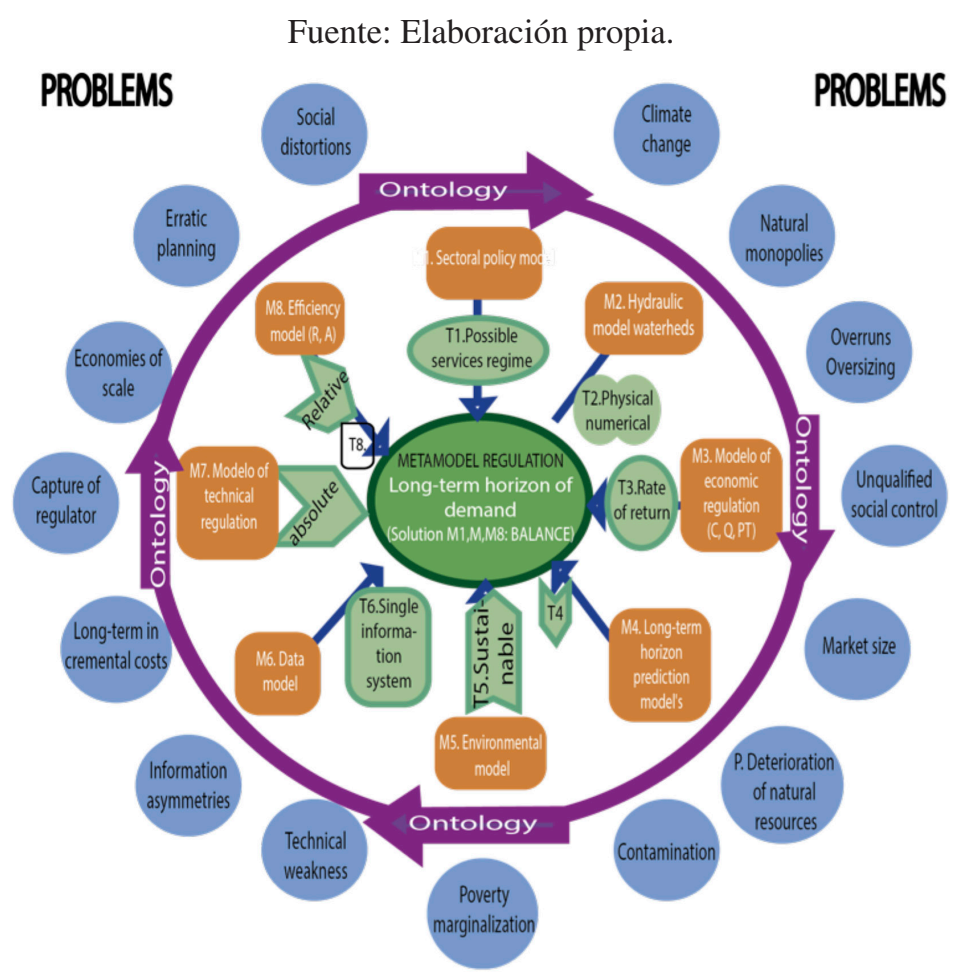

Figura 5. Esquema de la construcción de un meta modelo.

de las pérdidas técnicas considerando el número de conexiones, la presión promedio y el nivel de desempeño técnico de los prestadores. Así, por ejemplo, para Colombia se ha establecido un máximo anual x suscriptor de $54 \mathrm{~m}^{3}$ en el nivel de pérdidas técnicas. Como crítica, el IBM no ha sustentado este máximo.

c) En relación con las pérdidas comerciales no existen metodologías debidamente desarrolladas y probadas que permitan establecer los niveles aceptables de este tipo de pérdidas. Rangos entre el 25\% y el 50\% del nivel de pérdidas totales, corresponden a un nivel entre el 4,5\%-9,5\% del volumen de agua producida y todos ellos pueden ser sólo especulativos.

d) Índice de pérdidas aceptables.

\section{Subcaso de la EAAB (Bogotá)}

$\mathrm{Al}$ aplicar el modelo anterior a la empresa que suministra agua en Bogotá [67], observamos que el

Tabla 1. Síntesis de resultados EAAB.

\begin{tabular}{|l|c|l|}
\hline \multicolumn{1}{|c|}{ Variable } & Valor & \multicolumn{1}{c|}{ Observación } \\
\hline Total demanda & 15.073 .971 .374 & \\
\hline Tasa descuento & 0,1334 & \\
\hline Demanda corregida & 15.190 .557 .913 & \\
\hline VPD & 2.513 .313 .833 & \\
\hline Año & $2003-2046$ & \\
\hline VPD sin año cero o año base (1) & 2.205 .057 .609 & Contrario a Resolución CRA 287 de 2004 \\
\hline Estudio de la EAAB & 2.180 .168 .658 & Utilizado para obtener tarifas \\
\hline VPD con año base o año cero (2003) (3) & 2.513 .313 .833 & Conforme art. 30 de la Resolución CRA 287 de 2004. Dato correcto \\
\hline Diferencia (3) - (1) & 308.256 .224 & \\
\hline
\end{tabular}

Fuente: Elaboración propia. 
cálculo de la demanda corregida y el valor presente de la demanda en cada periodo sufre estos impactos ${ }^{3}$

Si bien es cierto que se aprecia una pequeña diferencia entre el dato obtenido por la empresa (sin año base) y el calculado en la investigación, dado que no es objeto del presente análisis, no nos detendremos en ella por considerarla marginal [67]. Lo relevante es que el VPD con el año base representaría que más de 300 millones de metros cúbicos se debieron incorporar a los cálculos de las tarifas de acueducto sin que así hubiera ocurrido. Se demuestra, entonces, una clara distorsión en la proyección de la demanda futura, con impactos en las tarifas y en la predicción inferior de la demanda futura, afectando a nuevas poblaciones que requerirán la dotación de agua potable.

\section{CONCLUSIONES}

El suministro de agua, en particular el destinado a consumo, se hace cada vez más complejo. Por problemas crecientes, como los de pérdida de ecosistemas, la contaminación, el cambio climático y la expansión urbana asimétrica, que impiden la renovación del agua, como bien natural.

Para buscar soluciones, que prevean en horizontes de largo plazo, desde la política sectorial y la prestación y control, se requiere de la articulación de modelos que, de manera integral, y bajo nuevos lenguajes, generen metamodelos de regulación, como el recién expuesto.

Colombia actualmente carece de elementos técnicos vitales en la regulación de la prestación del servicio público de acueducto, basados por ejemplo en la proyección de la demanda de agua potable, por tanto, el estudio constituye un aporte a la regulación y a la administración de otros servicios públicos.

Los modelos de referencia chilenos e ingleses, tienen como bases de su regulación principios que permiten estructurar esquemas sólidos y competentes, garantizando.

\section{AGRADECIMIENTOS}

Por el apoyo prestado y los recursos dispuestos para el presente paper, los autores manifiestan su

\footnotetext{
3 Ver Anexo $\mathrm{N}^{\mathrm{o}} 1$ Revisión del valor presente de la demanda para acueducto de la EAAB)
}

agradecimiento al Doctorado en Ingeniería de la Universidad Distrital Francisco José de Caldas, de Bogotá, Colombia.

\section{REFERENCIAS}

[1] A. Larsen and W.Gujer. "The concept of sustainable urban water management". Water Science and Technology. V. $35 \mathrm{~N}^{\circ}$ 9, pp. 3-10. 1997. DOI: 10.1016/S0273-1223(97)00179-0.

[2] Asamblea Nacional Constituyente "Constitución Política de Colombia". Artículos 365-370, 1991. Fecha de consulta: septiembre de 2017. URL: http://www. suin-juriscol.gov.co/viewDocument. asp?ruta $=$ Constitucion $/ 1687988$.

[3] J. Lizcano. "Investigación al Régimen Tarifario de Agua Potable y Saneamiento Básico, Bogotá: Fondo Editorial de la Universidad Distrital FJC". 2011.

[4] J. Salamanca, A. Gualy, G. Garcia, C. Gonzáles y E. Pedraza. "Regulación de Agua Potable y Saneamiento Básico". Regulación de Agua Potable y Saneamiento Básico. Vol. 17, pp. 1-165.2012. ISSN: 0123-370x.

[5] R. Palmer, A. Polebitski, L. Traynham, K. King and B. Enfield. "Review of Seattle's New Water Demand Model". University of Washington. 2004.

[6] Centro de Investigaciones Económicas. "Economía, principales conceptos". pp. 1-191. 2005. Fecha de consulta: septiembre de 2017. URL: https://idblegacy.iadb.org/res/ laresnetwork/files/pr242finaldraft.pdf.

[7] Superintendencia de Servicios Públicos, "Estratificación y Solidaridad". Supercifras. Vol. $1 \mathrm{~N}^{\circ} 1.1998$.

[8] Gobierno Nacional de Colombia. "Decretos 2220 de 1993, 1429 de 1995, 565 de 1996, 302 del 2000, 229 del 2002, 1013 del 2005, 057 del 2006 y 849 del 2002”. Gaceta Oficial, Bogotá.

[9] Ministerio de Desarrollo Económico. "Reglamento Técnico del Sector de APSB”. Mindesarrollo. Bogotá. 2000.

[10] Comisión de Regulación de Agua Potable y Saneamiento Básico. "Resumen participación ciudadana de las observaciones recibidas de las Resoluciones CRA 485, 486 y 487 de 2009”. Documento de Trabajo Proyecto General. CRA, Bogotá. 2014.

[11] P.p, R.a and M. Haase. "www.neon-project. org”. Neon Project. 2009. 
[12] IDEAM. "Informe Anual Sobre el Estado del Medio Ambiente y los Recursos Naturales Renovables en Colombia". Panamericana Formas e Impresos S.A. Bogotá. 2009.

[13] Comisión de Regulación de Agua Potable y Saneamiento Básico. "El Estado del Arte de la Regulación”. CRA, Bogotá. 2001.

[14] R. Lenton, A. Wright and K. Lewis. "Dignity, and Development, What will it Take? UN Millenium Project Task Force on Water and Sanitation", Final Report. Earthscan. London. 2005.

[15] J. De la Hoz. "Finanzas y gobierno de las corporaciones autónomas regionales del Caribe colombiano". pp. 1-58. 2010. Fecha de consulta: 04 de julio de 2018. URL: http://www.banrep.gov.co/sites/default/files/ publicaciones/archivos/DTSER-126.pdf.

[16] Banco Mundial, Andesco y Mindesarrollo. "Seminario Internacional de Regulación en Agua Potable y Saneamiento Básico". Arte Laser Publicidad Ltda. Bogotá. 2001.

[17] Superintendencia de Servicios Sanitarios "Regulación de tarifas". 2018. Fecha de consulta: 06 de julio de 2018. URL: http:// www.siss.gob.cl/586/w3-article-4336.html.

[18] Publications.parliament.uk "The Water White Paper". 2012-2013. Fecha de consulta: 06 de julio de 2018. URL: https://publications. parliament.uk/pa/cm201213/cmselect/ cmenvfru/374/374.pdf.

[19] M. Cave. "Independent Review of Competition in Water Markets". 2009. Fecha de consulta: 06 de julio de 2018. URL: https://assets. publishing.service.gov.uk/government/ uploads/system/uploads/attachment_data/ file/69462/cave-review-final-report.pdf.

[20] Gobierno Nacional de Colombia "LEYES 286 de 1996, 632 de 2000, 689 de 2001, 715 de 2001 y 812 de 2003, Ley 1151 del 2007 y Ley 1176 de 2007”. Bogotá.

[21] J. Blanco. "Integrated Water Resource Management in Colombia: Paralysis by Analysis. International Journal of Water Resources Development”. pp. 91-101. 2008. Fecha de consulta: 04 de julio de 2018. URL: https://www.tandfonline.com/doi/ full/10.1080/07900620701747686.

[22] A. Biswas, Cb. Tortajada and D. Rodriguez. "Water Quality Management in the Americas". Ed. Springer. 2006.
[23] L. Mantilla. "La autonomía de las CAR en la jurisprudencia constitucional de Colombia: Análisis de línea jurisprudencial”. pp. 1-18. 2015. Fecha de consulta: 04 de julio de 2018. URL:http://revistascientificas.cuc.edu.co/ index.php/juridicascuc/article/view/480/15.

[24] Superintendencia de Servicios Públicos Domiciliarios. "Informe Ejecutivo de Gestión Empresa de Acueducto y Alcantarillado de Bogotá”. Bogotá. 2014.

[25] Congreso Nacional de Colombia. "Régimen de los Servicios Públicos". 1994. Fecha de consulta: Septiembre de 2017. URL: http:// www.alcaldiabogota.gov.co/sisjur/normas/ Norma1.jsp?i=2752.

[26] J. Lizcano, S. Bolaños y R. Medina. "La ciencia del diseño para un metamodelo de regulación de agua potable y saneamiento básico". Redes de Ingeniería. Edición especial. pp.138-144. 2016. DOI: https:// doi.org/10.14483/2248762X.12002.

[27] Comisión de Regulación de Agua Potable y Saneamiento Básico "Resolución 12". 1995. Fecha de consulta: Septiembre de 2017. URL: http://www.minvivienda. gov.co/ResolucionesAgua/0012\%20-\%20 1995.pdf.

[28] Comisión de Regulación de Agua Potable y Saneamiento Básico "Resolución 18”. 1995. Fecha de consulta: Septiembre de 2017. URL: http://tramitesccu.cra.gov.co/normatividad/ admon1202/files/Resoluci\%C3\%B3n_18_ de_1995.pdf.

[29] Comisión de Regulación de Agua Potable y Saneamiento Básico "Resolución 74”. 1999. Fecha de consulta: Septiembre de 2017. URL: http://www.minvivienda.gov.co/ResolucionesAgua/0074\%20-\%201999.pdf.

[30] T. Coelli. "Frontier 4.1". Fecha de consulta: Febrero de 2015. URL: http://frontier.rforge.r-project.org/front41.html.

[31] Ministerio de Vivienda, Ciudad y Territorio. "Cien años de políticas habitacionales". Ed, Panamericana formas e impresos S.A., Bogotá, Colombia. ISBN: 978-958-57464-1-1. 2014.

[32] A.J. y Otros. "Consumo Básico. Regulación", CRA, Bogotá. 2000.

[33] C. Tecnico. "Documento Bases Regulatorias". CRA, Bogotá. 2003.

[34] H.R. "Exposición de motivos para el Régimen de los Servicios públicos”. Bogotá. 1992. 
[35] Congreso Nacional de Colombia. "Sistema Nacional Ambiental. Ley 99”. 1993. Fecha de consulta: septiembre de 2017. URL: http:// www.alcaldiabogota.gov.co/sisjur/normas/ Norma1.jsp?i=297.

[36] Congreso Nacional de Colombia "Ley 373 de Uso Racional y Eficiente del Agua". 1997. Fecha de consulta: Septiembre de 2017. URL: bhttp://www.alcaldiabogota. gov.co/sisjur/normas/Norma1.jsp?i=342.

[37] Comisión de Regulación de Agua Potable y Saneamiento Básico. "Segundo Periodo Tarifario de Acueducto, Resolución 287". 2004. Fecha de consulta: Septiembre de 2017. URL: http://www.alcaldiabogota.gov. co/sisjur/normas/Norma1.jsp?i=13956.

[38] Congreso Nacional de Colombia. "Régimen de los Servicios Públicos en Colombia, Ley 142”. 1994. Fecha de consulta: Septiembre de 2017. URL: http://www.alcaldiabogota. gov.co/sisjur/normas/Norma1.jsp?i=2752.

[39] J. Lizcano. "Nuevo modelo tarifario. Sector de Acueducto y Alcantarillado. Investigación 04-01”. Revista Superservicios. 2004.

[40] O. Bravo y M. Sanchez. "Gestión Integral de Riesgos". Ed Bravo \& Sánchez. Bogotá, Colombia. ISBN: 978958338873. 2014.

[41] Universidad de Cartagena. "Los Servicios Públicos Domiciliarios”. Cartagena, 1998.

[42] Ministerio de Desarrollo Económico "Definicion del nivel de complejidad y evaluación de la población, la dotación y la demanda de agua”. Bogotá. 2001.

[43] Ministerio de Desarrollo Economico. "Guia RAS-003 Planeamiento y diseño hidraulico de redes de distribucion de agua potable". Bogotá. 2001.

[44] J. Placido. "Bienes públicos e interés colectivo: la prestación del servicio público domiciliario de agua potable en Bogotá". 2012. Fecha de consulta: Septiembre de 2017. URL: http://bdigital.unal.edu.co/9129/1/ joseplacidosilvaruiz.2012.pdf.

[45] Universidad Externado de Colombia. "Derecho de Aguas". Bogotá. 2003.

[46] Asamblea Nacional Constituyente. "Constitución Política de Colombia”. 1991.

[47] Superintendencia de Servicios Públicos. "Guía para Auditorías Externas de Gestión y Resultados”. Bogotá. 2001.

[48] J. See, Q. Zhang and Y. Chen. "Statistical modeling of daily urban water consumption in Hong Kong: Trend, Changing patterns, and forecast". Water Resources Research. Vol. $46 \mathrm{~N}^{\circ} 3$, pp. 2-10. 2010. DOI: 10.1029/2009WR008147, 2010.

[49] Gobierno Nacional de Colombia. "Decreto Nacional 849". 2002. Fecha de consulta: Septiembre de 2017. URL: http://www. alcaldiabogota.gov.co/sisjur/normas/Norma1. jsp?i=6037.

[50] Gobierno Nacional de Colombia. "Decreto Nacional 1013". 2005. Fecha de consulta: Septiembre de 2017. URL: http://www. alcaldiabogota.gov.co/sisjur/normas/Norma1. jsp?i=16218.

[51] Gobierno Nacional de Colombia. "Decreto Nacional 057". 2006. Fecha de consulta: Septiembre de 2017. URL: http://www. alcaldiabogota.gov.co/sisjur/normas/Norma1. jsp?i=18774.

[52] S. Matias y Y. Agudelo. "Los servicios Públicos Domiciliarios en Colombia. Análisis Socio Jurídico". Bogotá, Colombia. 2001.

[53] M. Velilla. "Los Servicios Públicos como Instrumento de Solidaridad y Cohesión Social del Estado". Ed. Ediciones Jurídicas Gustavo Ibáñez C. Bogotá, Colombia. ISBN 9588192994. 2005.

[54] Comisión de Regulación de Agua Potable y Saneamiento Básico. "Metodología de Riesgo para Clasificación de Empresas. Resolución 315”. CRA, Bogotá. 2005.

[55] Superintendencia de Servicios Públicos Domiciliarios. "Acueducto, Alcantarillado y Aseo 1998-2001". Supercifras en $\mathrm{M}^{3}$. No 6 . 2002.

[56] Corte Constitucional. "Sentencia C-150", 2003. Fecha de consulta: Septiembre de 2017. URL: http://legal.legis.com.co/docu ment?obra=jurcol\&document=jurcol_7599 2041dea9f034e0430a010151f034.

[57] Ministerio de Vivienda, Ciudad y Territorio "100 años de políticas habitacionales. Para el séptimo Foro Urbano Mundial". Ed. Panamericana formas e impresos S.A. Bogotá, Colombia. ISBN: 978-958-57464-1-1. 2014.

[58] Comisión de Regulación de Agua Potable y Saneamiento Básico. "Consumo Básico". Regulacion No 7. Bogotá. 2001.

[59] Comisión de Regulación de Agua Potable y Saneamiento Básico. "Bases del Nuevo Marco Regulatorio para el Sector de Agua Potable y Saneamient Básico”. CRA, Bogota. 2001. 
[60] M. Rivera y M. Cruz. "Evaluación Económica de Proyectos. Análisis de Costo Mínimo de Expansión de Capacidades". ACODAL. $\mathrm{N}^{\circ}$ 227, pp. 13-30. 2011.

[61] S. Stones, E. Dzuray, D. Meisegeier and A. Dahlborg. "Decision-support tools for predicting the performance of water distribution and wastewater collection systems". National Risk Management Research Laboratory, U.S. Enviromental Protection Agency, Cincinnati, OH. USA, 2002.

[62] P.J. y A.P. "Los efectos ambientales del crecimiento urbano: vivienda y ecosistemas naturales en Bogotá entre 1950 y 2000”. Científica. No 6, pp. 125-145. 2004.

[63] Superintendencia de Servicios Públicos Domiciliarios. "Supercifras en usuarios y estratificación socioeconómica". Bogotá. 1988.
[64] P.M y A. Perez. "Herramientas para la toma de decisiones en la gestión de alcantarillados: propectivas de implementación en la EAAB". ACODAL. No 228, pp. 25-36. 2011.

[65] J. Lizcano. "El justiprecio de los servicios públicos domiciliarios en el sector de agua potable y saneamiento básico". Revista TECNOGESTION: Una Mirada al Ambiente. Vol. $1 \mathrm{~N}^{\circ}$ 1, pp. 53-57. 2004.

[66] Superintendencia de Servicios Públicos Domiciliarios. "Coberturas generales de acueducto y alcantarillado y coberturas mínimas de agua potable y alcantarillado". Bogota. 2007.

[67] J. Lizcano. "Distorsiones socioeconómicas del régimen tarifario de agua potable y saneamiento básico". Ed. Universidad Distrital Francisco Jose de Caldas. Bogotá, Colombia. ISBN: 978-958-8337-92-0. 2011. 